\title{
POLÍTICAS TRANSNACIONALES SOBRE APRENDIZAJE MÓVIL Y EDUCACIÓN: SELECCIÓN DE TEXTOS RELEVANTES
}

\author{
TRANSNATIONAL POLICIES ABOUT MOBILE LEARNING AND \\ EDUCATION: A SELECTION OF RELEVANT TEXTS
}

\author{
Narciso José López García \\ narcisojose.lopez@uclm.es
}

Facultad de Educación de Albacete

\section{RESUMEN}

El uso de las TIC en entornos educativos ha originado nuevas metodologías de enseñanza-aprendizaje. Una de ellas es el denominado «mobile learning» o aprendizaje móvil. Este trabajo profundiza en este concepto, recoge las directrices marcadas por tres de los organismos internacionales más representativos en política educativa (la UNESCO, la Unión Europea y el NMC) y se propone dar a conocer los planteamientos actuales sobre la implementación de aprendizaje móvil en los procesos de enseñanzaaprendizaje. Para ello, se ha utilizado el análisis de información como método de trabajo, en el que la descripción e interpretación de las ideas obtenidas se ha configurado como eje principal de la investigación. Por último, se presentan las conclusiones derivadas de este proceso de búsqueda, análisis y selección de textos en las que se pone de manifiesto el gran potencial de estas nuevas pedagogías.

PALABRAS CLAVE: aprendizaje móvil, políticas TIC, políticas educativas, dispositivos móviles.

\begin{abstract}
The use of ICT in educational environments has given rise to new teaching and learning methodologies. One of them is called mobile learning. This report goes in detail about this concept, collects the guidelines marked by three of the most representative international organizations in education politics (UNESCO, the European Union and the NMC) and aims to raise awareness of current approaches to the implementation of mobile learning in teaching and learning processes. For that end, the analysis of information has been used as a working method where the description and interpretation of the collected ideas was configured as the main point of the investigation. Finally, conclusions arising from this process of searching, analysis and texts selection of texts in which the huge potential of these new pedagogies based on the using of mobile devices at school is made clear are presented.
\end{abstract}

KEYWORDS: mobile learning, educational policies, ICT policies, mobile devices. 


\section{INTRODUCCIÓN}

En los últimos 15-20 años, el uso cotidiano de las tecnologías de la información y la comunicación por parte de niños y jóvenes ha modificado su estilo de vida, de pensamiento y de conocimiento, quedando perfectamente integrados en su día a día.

En el ámbito educativo, López García, De Moya, Cózar, Hernández \& Hernández (2016) señalan que la irrupción de teléfonos móviles, tabletas y otros aparatos portátiles, y su rápida extensión a todos los niveles, ha abierto un abanico de posibilidades para los entornos escolares de unas dimensiones incalculables e inesperadas, cambiando radicalmente el concepto de enseñanza-aprendizaje mediante TIC.

Sin embargo, esto supone, por un lado, una modificación de las normas de un buen número de centros educativos que prohíben o restringen su uso dentro de sus instalaciones $y$, por otro, un análisis profundo sobre la manera más efectiva de integrarlos en los procesos de enseñanza-aprendizaje; es imprescindible que el uso de estos dispositivos vaya acompañado de una metodología correcta y un modelo didáctico estructurado, flexible y bien definido que permita la consecución de verdaderos aprendizajes significativos (Fernández, 2016).

En este mismo sentido, Viñas (2016, p. 2) afirma que, para asegurarnos de que su integración sea un recurso efectivo, será necesario "rediseñar el modo de enseñar, aprender y evaluar". Sólo se convertirán en herramientas educativas de primer orden si su uso va acompañado de una buena planificación y de unas normas de utilización claras y bien definidas (Pajuelo, 2017).

Este enfoque pedagógico, denominado m-learning o aprendizaje móvil, se sustenta en los datos proporcionados por la UNESCO (2017), que sostiene que el $67 \%$ de la población mundial (cinco mil millones de personas) usa un dispositivo móvil habitualmente y que el $95 \%$ vive en una zona cubierta por, al menos, una red móvil básica 2G. Estos porcentajes abren las puertas a una realidad educativa diferente en la que los espacios de aprendizaje traspasan las paredes de la escuela, favoreciendo procesos de enseñanza-aprendizaje más contextuales, autónomos y ubicuos (Matos, Cacheiro, Sánchez \& González, 2012).

\subsection{M-learning o aprendizaje en movilidad}

El término m-learning (mobile learning) surge entre 1970 y 1980 (Stosic \& Bogdanovic, 2013) y Yot \& Marcelo (2015, p. 206) lo definen como:

Aquel aprendizaje que se desarrolla total o parcialmente utilizando dispositivos móviles. Supone la capacidad de aprender en todas partes y en todo momento, sin necesitar una conexión física permanente a las redes mediante smartphones, portátiles o tabletas.

De lo anterior se desprende que el m-learning es una nueva forma de aprendizaje que nunca termina, que posibilita la combinación entre movilidad geográfica y virtual permitiendo un aprendizaje contextualizado, accesible en cualquier momento y para cualquier información y que apunta a una nueva dimensión en los procesos educativos cuya característica fundamental radica en que puede atender 
necesidades urgentes de aprendizaje, ubicarse en escenarios móviles y posibilitar la interactividad en estos procesos (Montás, 2016).

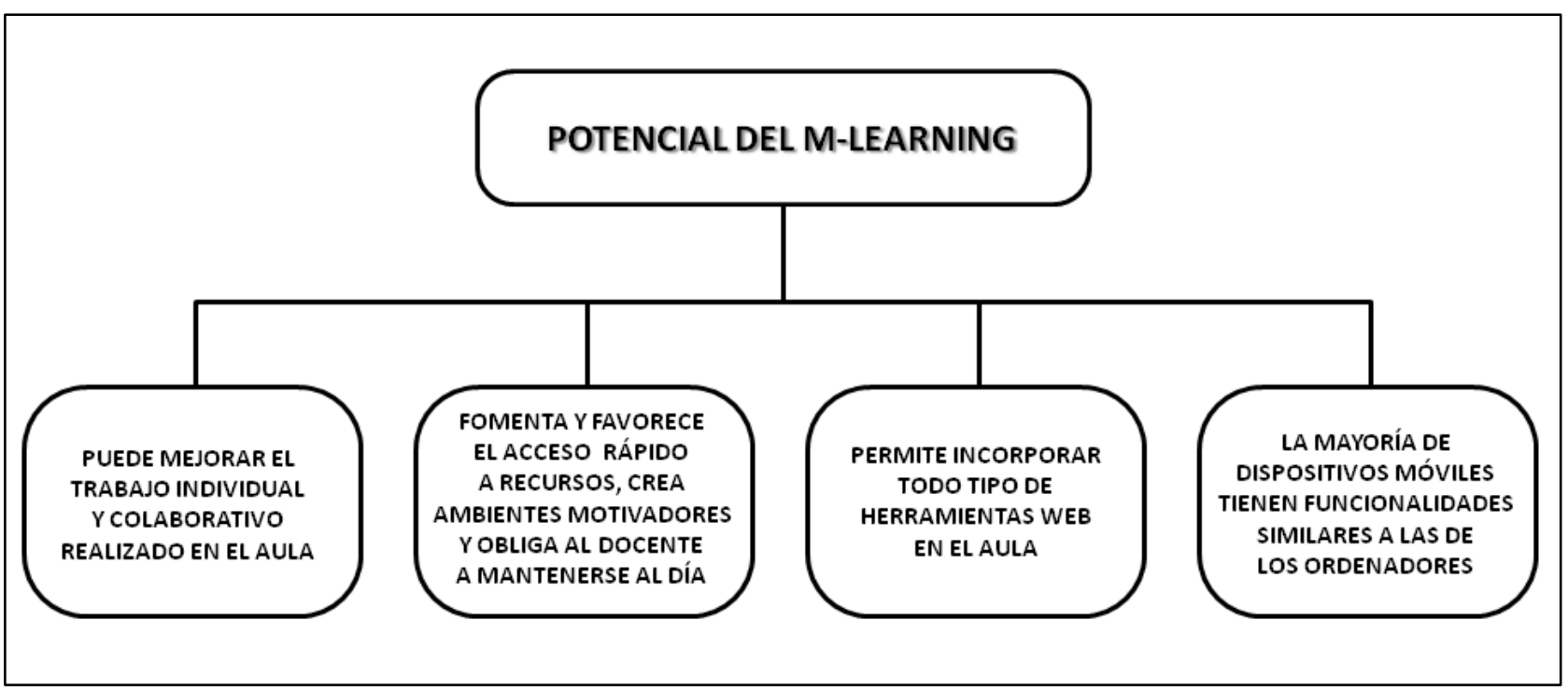

Figura 1. Potencial del $m$-learning aplicado a los procesos de enseñanza-aprendizaje de acuerdo con Santiago, Díez \& Navaridas (2014).

Sin embargo, el aprendizaje móvil está generando controversias entre los distintos sectores de la comunidad educativa, que no terminan de visualizar los beneficios del mismo y ponen en entredicho su utilidad educativa. Además, un buen porcentaje de docentes no termina de confiar en la influencia positiva de teléfonos inteligentes y tabletas en los procesos de enseñanza-aprendizaje, fundamentalmente porque no poseen la suficiente formación (Robledo, 2012). Surge, por tanto, la necesidad de programar y desarrollar actividades de formación que puedan garantizar la calidad de los proyectos que se están poniendo en marcha.

\subsection{UNESCO, Unión Europea y NMC}

Estas estrategias de formación e información están dirigidas a docentes, administraciones nacionales, regionales y locales con competencias en política educativa y a asociaciones de padres y madres de alumnos, y tienen la finalidad de capacitarlos para que la puesta en práctica de estos nuevos enfoques pedagógicos tenga plenas garantías de éxito. En este sentido, son varias las organizaciones internacionales que, conociendo el potencial de los dispositivos móviles en entornos educativos, han apostado por fomentar, facilitar y favorecer su implementación en los currículos escolares. Tres de estas organizaciones son: la UNESCO (Organización de las Naciones Unidas para la Educación, la Ciencia y la Cultura), la UE (Unión Europea) y el NMC (New Media Consortium) que, si bien podría ser muy arriesgado etiquetarlas como las más importantes instituciones dedicadas al desarrollo de políticas educativas, son las que han realizado los estudios más amplios e importantes relacionados con el binomio educación y TIC.

La UNESCO ha abordado extensamente la importancia de los dispositivos móviles en la sociedad actual y los beneficios de su uso en entornos educativos. Este interés ha 
originado la publicación de dieciséis documentos en los que se recogen iniciativas, proyectos, lecciones, directrices... destinadas tanto a los representantes políticos, como a los docentes y a otros agentes sociales relacionados con las tecnologías de la información y la comunicación.

Por su parte, la UE, desde la European Schoolnet ${ }^{1}$, colabora y participa para ayudar a los centros educativos a ser eficaces en el uso pedagógico de las TIC, ofreciendo al profesorado $y$ al alumnado las herramientas necesarias para conseguir una ciudadanía competente en habilidades digitales. En este empeño, ha publicado varios documentos en los que se exponen ejemplos concretos y directrices encaminadas a animar y a ayudar a los centros educativos a implementar los dispositivos móviles en sus proyectos de enseñanza-aprendizaje.

Finalmente, el NMC es un consorcio norteamericano de fabricantes de hardware, desarrolladores de software y editores (Apple Computer, Adobe Systems, Macromedia y Sony) que, desde el año 2004, publica el Informe Horizon, un ambicioso proyecto de investigación en el que se identifican tecnologías, desafíos y tendencias relacionadas con la innovación educativa y su implantación a corto, medio y largo plazos en las diferentes etapas educativas.

\section{METODOLOGÍA}

Este estudio se ha centrado en buscar, analizar, seleccionar y reseñar los documentos fundamentales sobre el aprendizaje móvil publicados por estas tres organizaciones internacionales.

El método de trabajo utilizado ha sido el análisis de información, entendido este como la forma de investigar cuyo objetivo consiste en captar, evaluar, seleccionar y sintetizar los mensajes contenidos en una serie de documentos (Dulzaides \& Molina, 2004). De esta forma, la descripción y la interpretación de los textos a estudio se ha convertido en el eje principal de la investigación (Bardin, 2002).

Para ello, se ha realizado una búsqueda exhaustiva de textos y, una vez analizados, se han seleccionado aquellos que mejor recogen las políticas trasnacionales sobre la inclusión del aprendizaje móvil en los procesos de enseñanza-aprendizaje.

Debido a las limitaciones de extensión, en este artículo se expone una breve reseña de cada uno de los informes seleccionados con la idea de facilitar una aproximación cognitiva del lector al contenido de las fuentes originales (Peña \& Pirela, 2007).

\section{ANÁLISIS DE TEXTOS RELEVANTES SOBRE EL APRENDIZAJE MÓVIL EN ENTORNOS EDUCATIVOS}

\subsection{La serie de documentos de la UNESCO sobre el aprendizaje móvil}

Entre 2012 y 2017, la UNESCO ha publicado dieciséis documentos de trabajo sobre aprendizaje móvil divididos en cuatro grandes secciones. Con ellos, se propone

\footnotetext{
${ }^{1}$ Red formada por 34 Ministerios Europeos de Educación (incluidos los 28 estados miembros de la UE) cuyo objetivo es llevar a cabo proyectos que favorezcan y faciliten la innovación en la enseñanza. 
lograr una mayor comprensión de cómo las tecnologías móviles pueden ser utilizadas para mejorar el acceso, la equidad y la calidad de la educación en todo el mundo. Además, proporciona a los responsables políticos, a los docentes y a otros agentes interesados un instrumento fundamental para aprovechar la tecnología móvil en el perfeccionamiento del aprendizaje.

\begin{tabular}{|c|c|}
\hline $\begin{array}{c}\text { SECCIÓN } 1 \\
\text { Iniciativas ilustrativas e implicaciones } \\
\text { políticas } \\
\text { (publicados en 2012) }\end{array}$ & $\begin{array}{l}\text { Activando el aprendizaje móvil en África y Medio Oriente } \\
\text { Activando el aprendizaje móvil en América Latina } \\
\text { Activando el aprendizaje móvil en América del Norte } \\
\text { Activando el aprendizaje móvil en Asia } \\
\text { Activando el aprendizaje móvil en Europa } \\
\text { Activando el aprendizaje móvil: Temas globales }\end{array}$ \\
\hline $\begin{array}{c}\text { SECCIÓN } 2 \\
\text { Análisis del potencial de las } \\
\text { tecnologías móviles para apoyar a los } \\
\text { docentes y mejorar sus prácticas } \\
\text { (publicados en 2012) }\end{array}$ & $\begin{array}{l}\text { Aprendizaje móvil para docentes en África y Medio Oriente } \\
\text { Aprendizaje móvil para docentes en América Latina } \\
\text { Aprendizaje móvil para docentes en América del Norte } \\
\text { Aprendizaje móvil para docentes en Asia } \\
\text { Aprendizaje móvil para docentes en Europa } \\
\text { Aprendizaje móvil para docentes: Temas globales }\end{array}$ \\
\hline $\begin{array}{c}\text { SECCIÓN } 3 \\
\text { Cuestiones clave e implicaciones } \\
\text { para la planificación y la formulación } \\
\text { de políticas } \\
\text { (publicados en 2013) }\end{array}$ & $\begin{array}{l}\text { Aprendizaje móvil y políticas. Cuestiones clave } \\
\text { El futuro del aprendizaje móvil. Implicaciones para la } \\
\text { planificación y la formulación de políticas }\end{array}$ \\
\hline $\begin{array}{l}\text { SECCIÓN } 4 \\
\text { Otros documentos }\end{array}$ & $\begin{array}{l}\text { Directrices para las políticas de aprendizaje móvil (2013) } \\
\text { Supporting teachers with mobile technology. Lessons drawn } \\
\text { from UNESCO projects in Mexico, Nigeria, Pakistan and } \\
\text { Senegal (2017) }\end{array}$ \\
\hline
\end{tabular}

Tabla 1. Serie de documentos de trabajo de la UNESCO sobre aprendizaje móvil.

Aunque en este artículo solo se desgrana el contenido de los documentos globales, es necesario anotar que los otros informes examinan los esfuerzos llevados a cabo en las diferentes regiones del mundo, ofrecen algunas iniciativas ilustrativas del aprendizaje móvil y sus implicaciones en las políticas educativas de las zonas estudiadas, analizan las posibilidades de las tecnologías móviles como herramientas de apoyo a la práctica docente y exponen diversas lecciones destinadas a los responsables políticos y a otros agentes sociales interesados en aprovechar el potencial de los dispositivos móviles en la educación.

\subsubsection{Activando el aprendizaje móvil: Temas globales. Políticas}


Este texto presenta un resumen de los resultados e ideas fundamentales publicadas en los cinco estudios regionales que completan la Sección 1. En él se confirma la omnipresencia de los teléfonos móviles y su papel como representantes interactivos mayoritarios de las TIC en el mundo y se expone su potencial en la mejora de la educación. Por último, se definen las estrategias principales sobre la activación del aprendizaje móvil.

Actualmente, más del $70 \%$ de las suscripciones de todo el mundo a planes de telefonía móvil están en los países en desarrollo

La telefonía móvil facilita el acceso a una educación de alta calidad

Diferentes proyectos y experiencias educativas llevadas a cabo en los últimos veinte años ponen de manifiesto el valor educativo y las oportunidades de las tecnologías móviles

La puesta en práctica de proyectos educativos mediante aprendizaje móvil requiere planificación, persistencia y una fuerte dosis de pruebas y errores.

Existe una postura demasiado extendida de prohibir el uso de los teléfonos móviles en los centros educativos, aunque lo más adecuado sería posicionar a las escuelas como instituciones que pueden enseñar a los alumnos a usar esta tecnología con responsabilidad

El acceso móvil por sí mismo ni asegura ni fomenta el aprendizaje. Es necesario enseñar a convertir un dispositivo móvil en una herramienta de aprendizaje

El aprendizaje móvil conlleva el potencial de ayudar a las personas que históricamente han carecido de oportunidades educativas

Dos de los mayores inconvenientes encontrados en el uso masivo de la telefonía móvil son los elevados costes en algunas partes del mundo y los obstáculos técnicos para construir plataformas capaces de optimizar el contenido para una mayoría de dispositivos sin que la disparidad de marcas, modelos, calidades, procesadores o sistemas operativos sea un problema

Tabla 2. Ideas principales desarrolladas en el informe Activando el aprendizaje móvil: Temas globales.

\subsubsection{Aprendizaje móvil para docentes: Temas globales}

Este documento recoge la información desarrollada en los cinco estudios relacionados con el aprendizaje móvil para docentes que completan la Sección 2. Estos están organizados de acuerdo a parámetros geográficos y describen un importante número de proyectos de innovación, desde iniciativas para facilitar la comunicación entre docentes y alumnos, hasta propuestas en las que la telefonía móvil se utiliza para fomentar la participación y la interactividad entre grupos numerosos de estudiantes. 


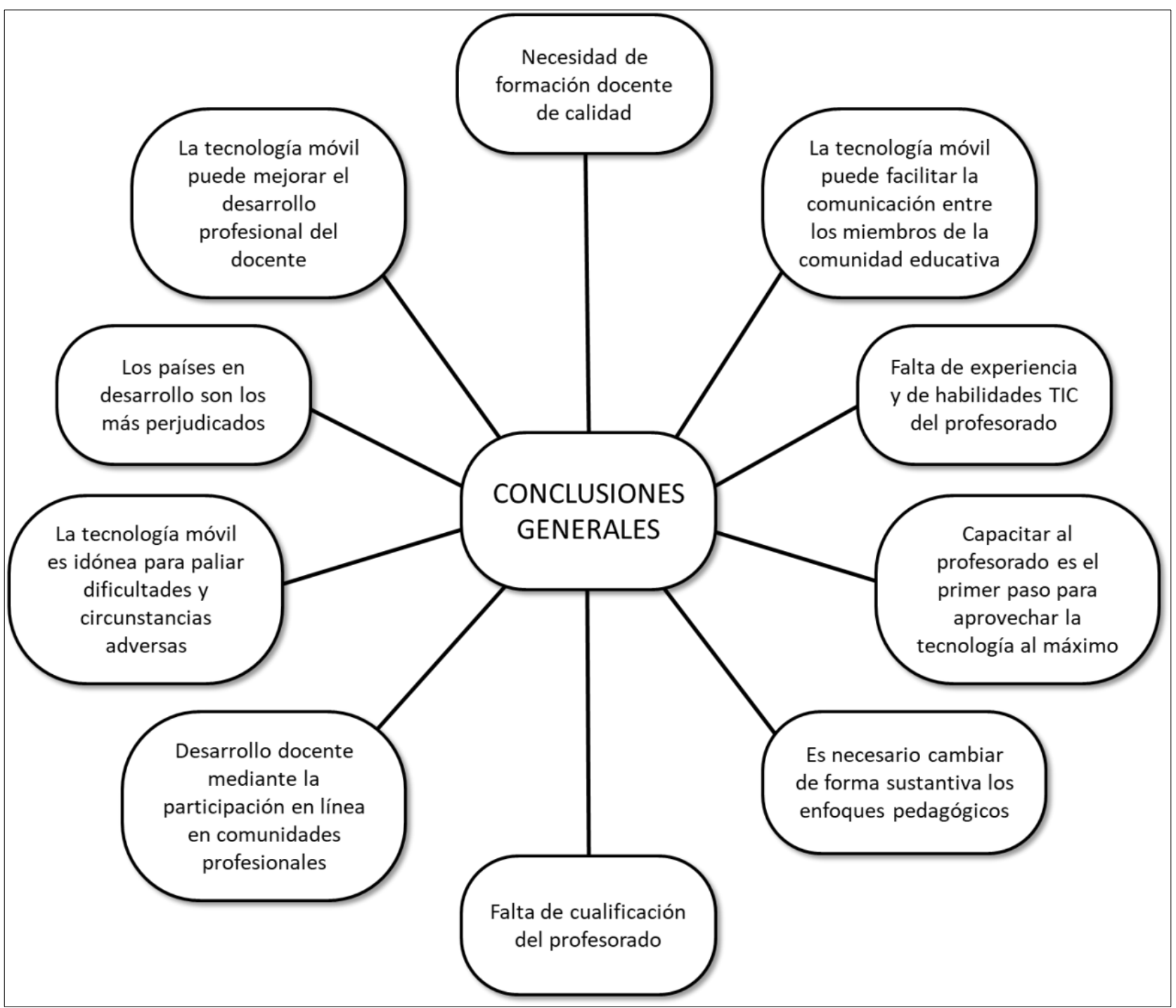

Figura 2. Conclusiones generales desarrolladas en este informe.

\subsubsection{Aprendizaje móvil y políticas. Cuestiones clave}

Este texto suministra a los responsables políticos una mejor comprensión del aprendizaje móvil y sus contextos, ofrece las definiciones de dispositivo móvil y de aprendizaje móvil y desarrolla diferentes consideraciones encaminadas a relacionar las políticas del aprendizaje móvil con las TIC ya incorporadas a las políticas educativas. Además, presenta los problemas más significativos derivados de su implementación en los procesos educativos así como los principios rectores que deberían seguirse a la hora de establecer políticas de aprendizaje móvil.

Por último, define los principios rectores a tener en cuenta a la hora de crear o revisar políticas educativas sobre aprendizaje en movilidad. 


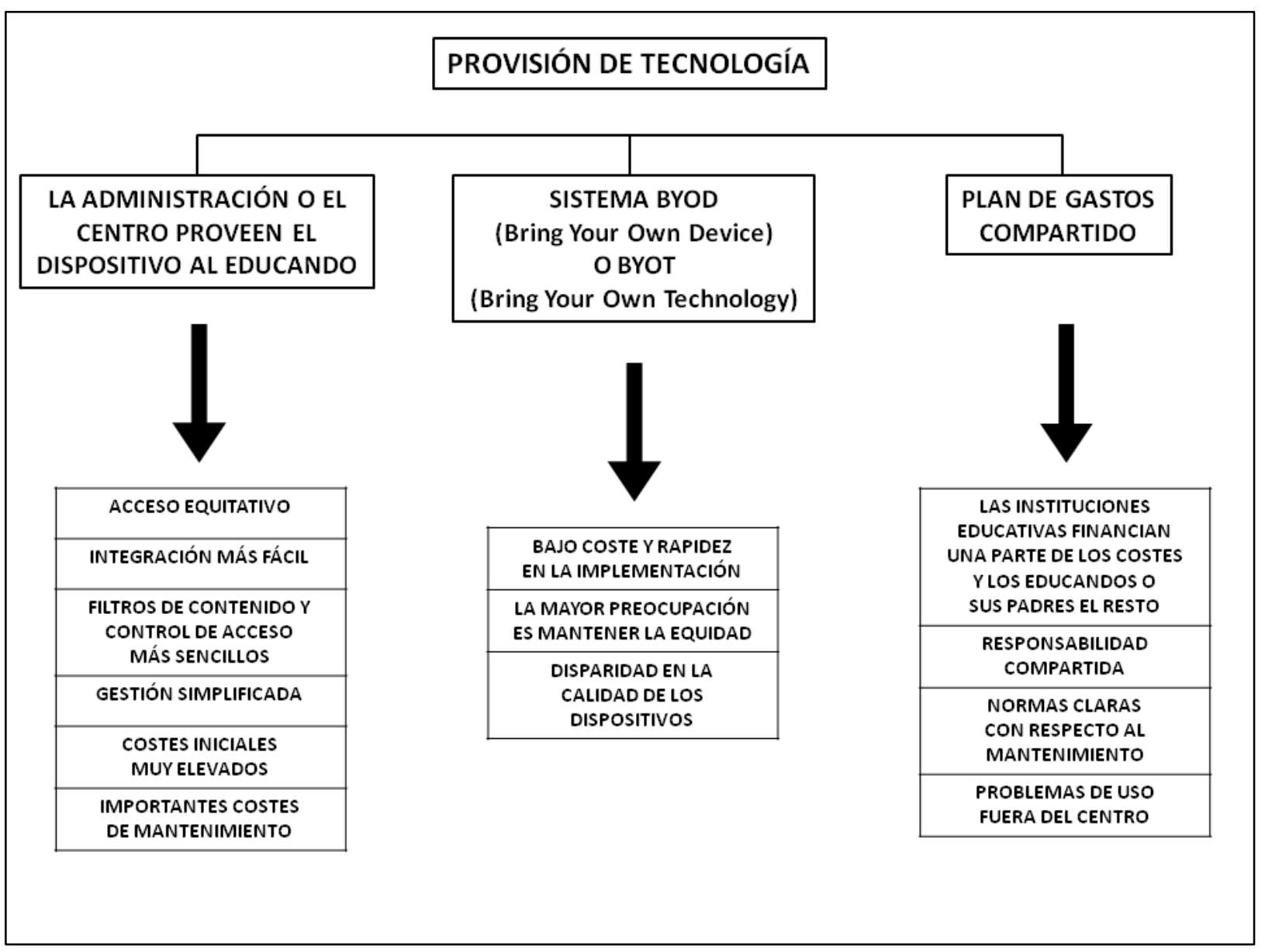

Figura 3. Modelos de provisión de la tecnología en los centros educativos.

\subsubsection{El futuro del aprendizaje móvil. Implicaciones para la planificación y la formulación de políticas}

La finalidad última de este documento es orientar a los responsables de formular políticas educativas en el camino de la transformación educativa hacia la plena inclusión del aprendizaje móvil en el aula, poniendo sobre la mesa cuestiones que marcarán el futuro, a corto y medio plazo, relacionadas con estos nuevos métodos de enseñanza y aprendizaje. Por otro lado, se exponen los grandes retos a afrontar en los próximos quince años para que el aprendizaje móvil se incorpore a los sistemas educativos ordinarios y tenga una incidencia real a escala mundial. Finalmente, se presenta una serie de preguntas a las que será conveniente dar respuesta para que el aprendizaje móvil pueda pasar de ser un ámbito de innovación desigual y dispersa a configurarse como una fuerza dinámica de impacto en la educación mundial. 


\section{PREGUNTAS PARA EL FUTURO}

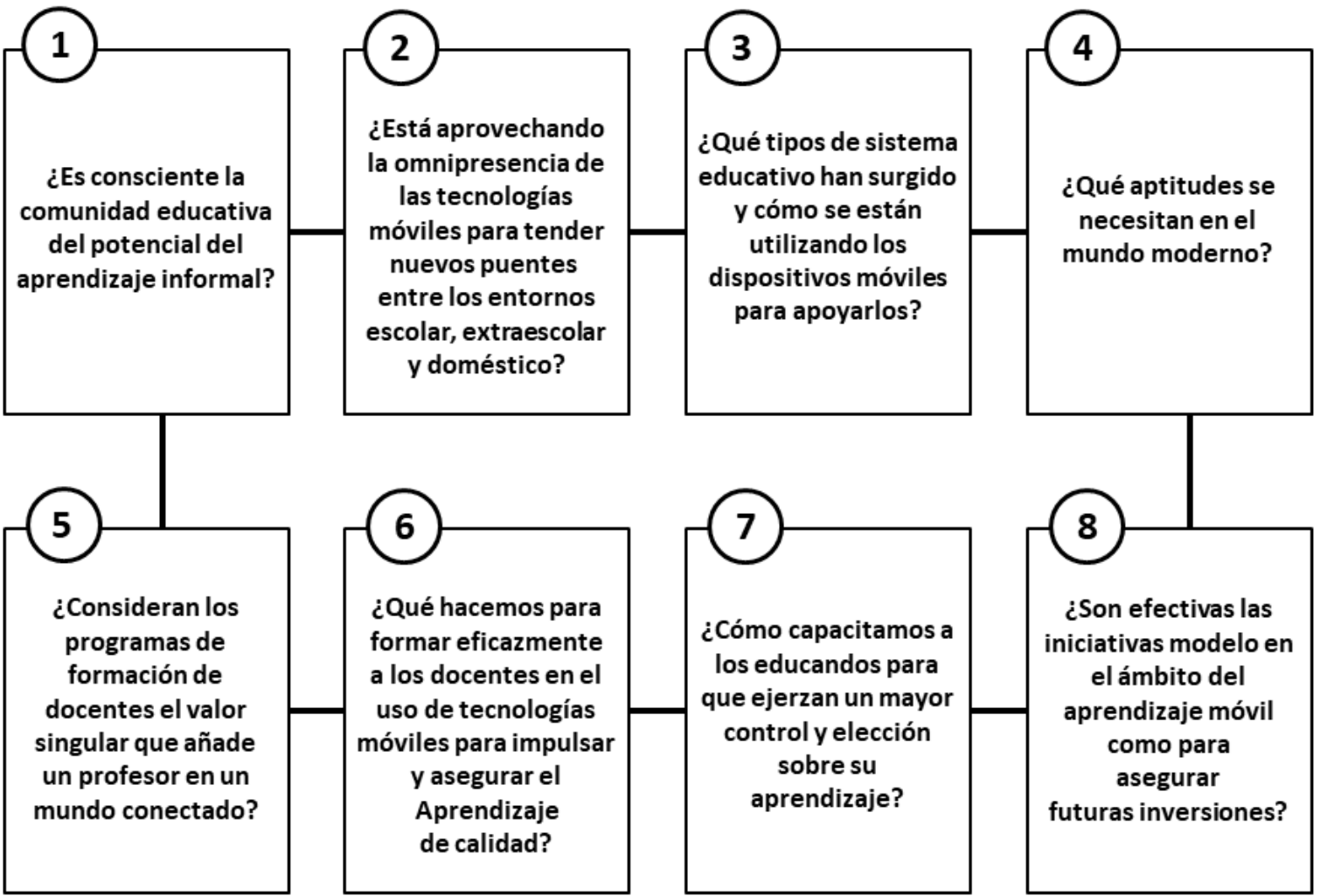

Figura 4. Cuestiones clave para que el aprendizaje móvil se convierta en fuerza dinámica de impacto.

\subsubsection{Directrices para las políticas de aprendizaje móvil}

Estas directrices tienen por objeto ayudar a los responsables de formular políticas a entender mejor en qué consiste el aprendizaje móvil y cómo pueden aprovecharse sus ventajas para impulsar la Educación para Todos. Por otro lado, se presenta una serie de alegatos con los que se intentan dar a conocer las oportunidades educativas que ofrece el aprendizaje en movilidad.

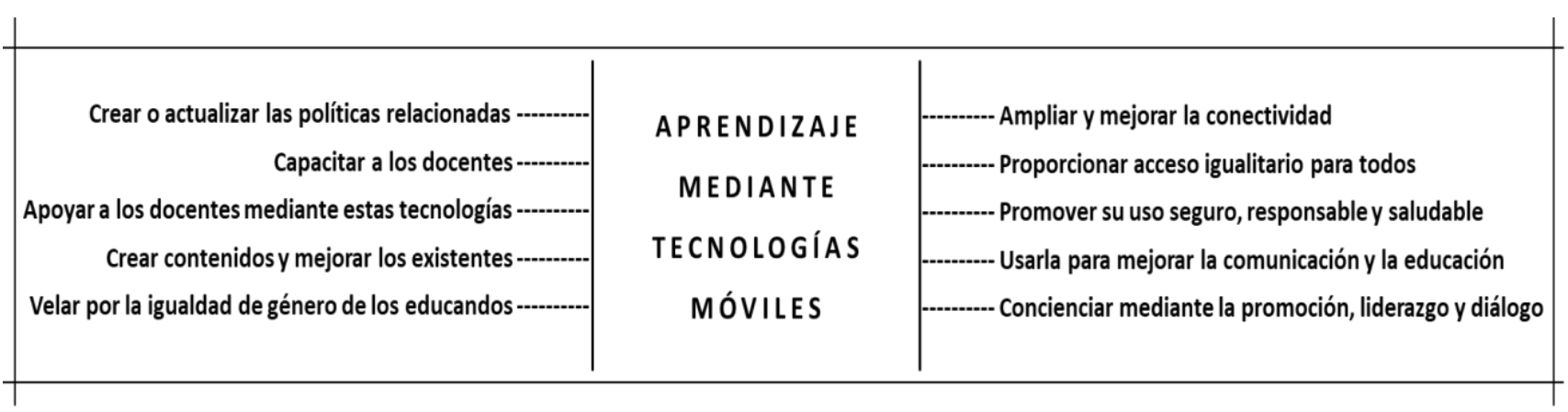

Figura 5. Directrices de la UNESCO para las políticas de aprendizaje móvil. 


\subsubsection{Supporting teachers with mobile technology. Lessons drawn from UNESCO projects in Mexico, Nigeria, Pakistan and Senegal}

En éste se exponen los diferentes motivos por los que los docentes deberían implementar los teléfonos móviles en el aula. A su vez, resume las ideas fundamentales obtenidas de los proyectos llevados a cabo en Méjico, Nigeria, Pakistán y Senegal, ofrece una serie de recomendaciones en relación a los resultados obtenidos y marca unas sencillas directrices a tener en cuenta para cumplir los objetivos marcados en la Agenda de Educación 2030 de la UNESCO.

\subsection{Documentos relevantes sobre aprendizaje móvil financiados por la Unión Europea}

La Unión Europea, en consonancia con las recomendaciones y directrices establecidas por la UNESCO, ha colaborado en la financiación y publicación de diferentes documentos relacionados directamente con la educación. De todos ellos, son dos los informes que se han dedicado al aprendizaje móvil, ofreciendo un material de máximo interés para la implementación de los dispositivos móviles en los procesos de enseñanza y aprendizaje de los países que configuran la European Schoolnet. En ellos, se anima y se ayuda a los estados miembros interesados en introducir el aprendizaje móvil en sus políticas educativas mediante la presentación de proyectos, recomendaciones y directrices específicas.

\subsubsection{Mobile learning and cloud services beyond the textbook. Recommendations and guidelines for policy makers}

Este texto desarrolla tres estudios regionales, expone las prioridades y desafíos más importantes surgidos de los mismos y define siete recomendaciones y líneas de actuación a seguir en el desarrollo de políticas educativas relacionadas con la implementación de dispositivos móviles y el diseño de estrategias para el aprendizaje en movilidad que deberían tener en cuenta los responsables políticos de los países europeos. 


\begin{tabular}{|c|l|}
\hline \multicolumn{2}{|c|}{ Recomendaciones y líneas de acción } \\
\hline $\begin{array}{c}\text { La educación más allá } \\
\text { del libro de texto }\end{array}$ & $\begin{array}{l}\text { Los responsables de las políticas educativas, en colaboración con los } \\
\text { desarrolladores de software, editores, docentes y estudiantes deberían } \\
\text { desarrollar estrategias que respalden la creación de recursos educativos } \\
\text { digitales innovadores para los centros escolares }\end{array}$ \\
\hline $\begin{array}{c}\text { Educación basada en la } \\
\text { equidad }\end{array}$ & $\begin{array}{l}\text { Hay que garantizar que todos los estudiantes tengan acceso a las } \\
\text { metodologías de enseñanza y aprendizaje basadas en el uso de las TIC, } \\
\text { así como que todos los docentes sean digitalmente competentes }\end{array}$ \\
\hline Visión & $\begin{array}{l}\text { Puesta en marcha de mecanismos que muestren una visión clara de la } \\
\text { importancia de las TIC en la educación, especialmente para los países que } \\
\text { carecen de estrategias digitales en sus sistemas educativos. Además, hay } \\
\text { que fomentar y transmitir la idea de que los dispositivos móviles se } \\
\text { pueden implementar en el aula de manera efectiva }\end{array}$ \\
\hline $\begin{array}{c}\text { Nuevos modelos de } \\
\text { compra }\end{array}$ & $\begin{array}{l}\text { Modelos de adquisición de artefactos digitales que pongan en valor que el } \\
\text { aprendizaje se lleva a cabo cada vez más fuera de la escuela }\end{array}$ \\
\hline $\begin{array}{c}\text { Asociaciones públicas- } \\
\text { privadas }\end{array}$ & $\begin{array}{l}\text { Es necesario explotar más activamente la idea de que los modelos de } \\
\text { asociaciones público-privadas pueden apoyar enfoques innovadores de } \\
\text { implementación de las TIC en el aula }\end{array}$ \\
\hline $\begin{array}{c}\text { Prohibiciones } \\
\text { relacionadas con el uso } \\
\text { de la telefonía móvil }\end{array}$ & $\begin{array}{l}\text { Hay que cambiar las políticas que prohiben el uso de teléfonos móviles en } \\
\text { las escuelas mediante el desarrollo de pautas y políticas claras }\end{array}$ \\
\hline $\begin{array}{c}\text { Desarrollo profesional y } \\
\text { formación permanente } \\
\text { del profesorado }\end{array}$ & $\begin{array}{l}\text { Hay que aumentar los esfuerzos dirigidos a la formación continua de los } \\
\text { docentes, proponer unos niveles mínimos en competencias digitales y } \\
\text { configurar estrategias potentes con las que formarlos }\end{array}$ \\
\hline
\end{tabular}

Tabla 3. Recomendaciones y líneas de acción a tener en cuenta en el desarrollo de políticas relacionadas con los dispositivos móviles y el aprendizaje móvil en los procesos de enseñanzaaprendizaje.

\subsubsection{Designing the future classroom. BYOD (Bring Your Own Device). A guide for school leaders}

En éste se ofrece a profesores, equipos directivos, administraciones educativas y otros sectores relacionados con la educación información exhaustiva sobre las tendencias actuales, opciones y ejemplos concretos de centros educativos europeos relacionados con la implementación del modelo BYOD. Además, presenta diversas experiencias realizadas en otras partes del mundo en las que este modelo se ha convertido en uno de los métodos más significativos en el uso y en la integración de las tecnologías y del aprendizaje móvil en los procesos de enseñanza y aprendizaje.

Para ello, presenta una serie de requisitos que deben reunir los centros a la hora de implementar los dispositivos móviles mediante el sistema BYOD y desarrolla una 
serie de beneficios y de riesgos a tener en cuenta a la hora de trabajar con ellos en el aula.

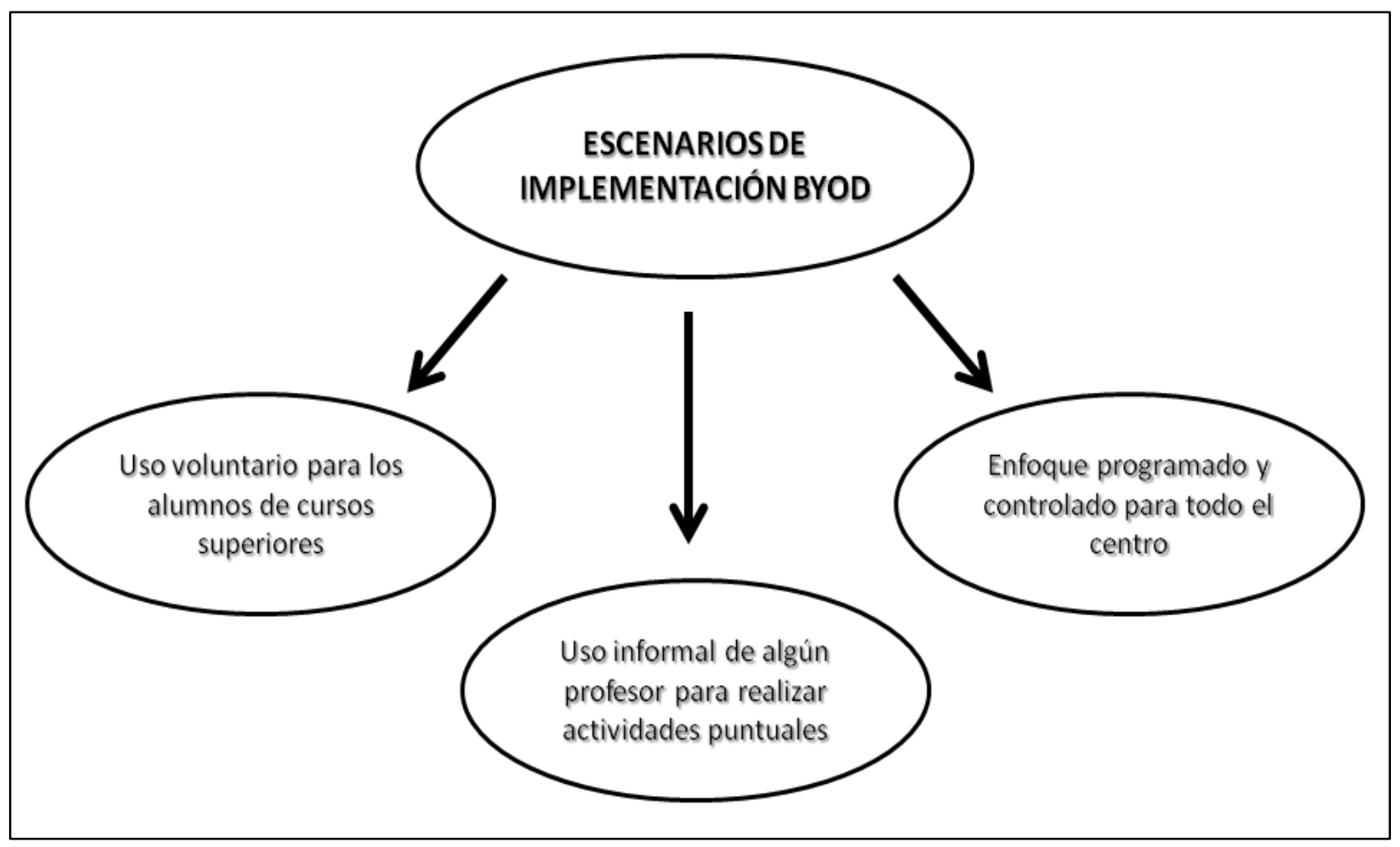

Figura 6. Posibles escenarios de implementación del método BYOD en centros escolares.

\subsection{Los informes Horizon del NMC}

Estos informes, elaborados por expertos internacionales, comienzan a publicarse en 2004 y su función principal es la de identificar y describir las tendencias más significativas, los desafíos y las tecnologías emergentes más relevantes que marcarán las acciones educativas a cinco años vista. 


\begin{tabular}{|c|c|c|}
\hline $\begin{array}{c}\text { Año de } \\
\text { publicación }\end{array}$ & Tecnología & Plazo de adopción \\
\hline 2004 & \multicolumn{2}{|c|}{ No hay referencias explícitas } \\
\hline 2005 & \multicolumn{2}{|c|}{ No hay referencias explícitas } \\
\hline 2006 & Teléfonos de bolsillo & De 4 a 5 años \\
\hline 2007 & Telefonía móvil & De 2 a 3 años \\
\hline 2008 & Banda ancha móvil & 1 año o menos \\
\hline 2009 & Móviles & 1 año o menos \\
\hline 2010 & Informática móvil & 1 año o menos \\
\hline 2011 & Informática móvil & 1 año o menos \\
\hline 2012 & Apps para dispositivos móviles & 1 año o menos o menos \\
\hline 2013 & Tabletas & De 4 a 5 años \\
\hline 2014 & Tabletas & No hay referencias explícitas \\
\hline 2015 & Tecnología portátil & 1 año o menos menos \\
\hline 2016 & No hay referencias explícitas & 1 año o menos \\
\hline 2017 & BYOD o BYOT & 1 año o menos \\
\hline
\end{tabular}

Tabla 4. Presencia de las tecnologías móviles en los informes Horizon de Educación Superior

\begin{tabular}{|c|c|c|}
\hline $\begin{array}{c}\text { Año de } \\
\text { publicación }\end{array}$ & Tecnología & Plazo de adopción \\
\hline 2009 & Móviles & De 2 a 3 años \\
\hline 2010 & Móviles & De 2 a 3 años \\
\hline 2011 & Informática móvil & 1 año o menos \\
\hline 2012 & Apps para dispositivos móviles & 1 año o menos \\
\hline 2013 & Tabletas & 1 año o menos \\
\hline 2014 & Aprendizaje móvil & 1 año o menos \\
\hline 2015 & BYOD o BYOT & 1 año o menos \\
\hline 2016 & BYOD o BYOT & 1 año o menos \\
\hline 2017 & Aprendizaje en línea & No hay referencias explícitas \\
\hline
\end{tabular}

Tabla 5. Presencia de las tecnologías móviles en los informes Horizon de Primaria y Secundaria

En la actualidad, los informes Horizon son textos de referencia para expertos y profesionales de la educación y las TIC y se divulgan en formato digital y en diferentes idiomas ${ }^{2}$.

\subsection{Posturas discrepantes y estudios desfavorables}

Los documentos anteriores se centran en exponer y avalar los beneficios que el aprendizaje móvil puede aportar a los procesos educativos y en proponer diferentes vías para su implementación en las aulas. Sin embargo, carecen de una reflexión

\footnotetext{
${ }^{2}$ Más información y acceso a los informes a texto completo en: https://www.nmc.org/nmc-horizon/ 
profunda sobre aquellos aspectos que pueden resultar nocivos en dicha implementación.

En los últimos años, están apareciendo diferentes opiniones que ponen en entredicho las bondades del m-learning y que desaconsejan, a priori, su uso como herramienta educativa. Así, expertos como Mosquera (2018) y Babín (en Rubio, 2018) reconocen que existen casos de uso irresponsable de estas tecnologías que pueden provocar situaciones de riesgo como, por ejemplo, el aumento del ciberbullying.

Además, se han detectado otros posibles aspectos negativos tales como el fomento del consumismo y del phubbing ${ }^{3}$, la distorsión del ritmo normal de la clase y la falta de disciplina, la limitación de la imaginación, el deterioro del funcionamiento cognitivo, el uso inadecuado e incorrecto del lenguaje, incluso la aparición de daños cervicales, estrés visual, insomnio, obesidad infantil, sedentarismo, dependencia o adicción a estos artefactos.

Esta corriente escéptica pone de manifiesto la necesidad urgente de llevar a cabo estudios más profundos con los que depurar todo tipo de dudas sobre las ventajas e inconvenientes reales del aprendizaje móvil, y abre nuevas vías de investigación a futuros trabajos en los que aquellos factores adversos que pueden aparecer, o que están apareciendo, en los proyectos actuales y venideros tengan mayor presencia y divulgación.

\section{CONCLUSIONES}

La implementación de los dispositivos móviles y del aprendizaje móvil en los entornos educativos es una realidad. El hecho de que un altísimo porcentaje de estudiantes y docentes tengan y estén acostumbrados a utilizar dispositivos móviles ha impulsado la práctica de nuevas estrategias curriculares en las que la búsqueda y consulta de información mediante estos artefactos se ha convertido en una rutina habitual, tanto dentro como fuera del aula. Sin embargo, la comunidad educativa ha manifestado sus dudas y preocupaciones sobre las ventajas que este modelo puede ofrecer en los procesos de enseñanza-aprendizaje.

No cabe duda de que el uso de teléfonos inteligentes, tabletas y otros dispositivos móviles requiere un rediseño de los modos de enseñanza, aprendizaje y evaluación, así como la concreción de metodologías claras, flexibles y bien definidas. Además, la puesta en marcha de diferentes estrategias de formación e información de todos y cada uno de los componentes de la comunidad educativa ha pasado a ser una necesidad imperiosa sin la cual muchos de los proyectos que se están llevando podrían llegar a sufrir un perjuicio muy significativo.

En este sentido, la UNESCO, la UE y el NMC se han propuesto dar a conocer a docentes, instituciones educativas, familias y empresas relacionadas con el uso de las tecnologías en contextos educativos los beneficios de la implementación de las

\footnotetext{
${ }^{3}$ Mosquera (2018) define el phubbing como la acción de prestar más atención al móvil que a las
} personas que nos acompañan. 
técnicas y tendencias más actuales en procesos de enseñanza-aprendizaje, así como los resultados obtenidos en situaciones reales mediante la realización de proyectos y la publicación de estudios de amplio espectro.

En los documentos reseñados se recogen diferentes escenarios de implementación del aprendizaje móvil en el aula, ventajas e inconvenientes que se pueden encontrar en el proceso, riesgos y retos a los que nos enfrentamos, así como diferentes experiencias y opiniones de centros educativos, expertos y profesionales de la educación que ponen de manifiesto el importante papel de los dispositivos móviles en contextos educativos. Además, con su análisis se ha podido comprobar la homogeneidad existente entre las tres organizaciones presentadas, hecho que favorece sobremanera el trabajo futuro y el cumplimiento de directrices y objetivos marcados.

Las posibilidades que nos ofrecen estos dispositivos son innumerables, tanto que sería un error hacer de la educación un mero espectador al margen de las opciones que se abren con la incorporación de los mismos en los procesos formativos. Se hace necesario, por tanto, definir procesos de análisis, valoración y reestructuración que permitan su implementación. Sin embargo, es necesario tener en cuenta aquellas voces discrepantes que ponen de manifiesto la existencia de riesgos potenciales. Solo de esta manera los currículos serán capaces de proporcionar al alumnado aprendizajes más dinámicos, atractivos y actualizados, dentro de entornos seguros, acordes con la era tecnológica en la que están inmersos.

\section{REFERENCIAS BIBLIOGRÁFICAS}

Ayre, J. (2015). Designing the future classroom. BYOD (Bring Your Own Device). A guide for school leaders. Brussels: European Schoolnet.

Bardin, L. (2002). Análisis de contenido. Tres Cantos (Madrid): Akal.

Dulzaides, M.E. \& Molina, A.M. (2004). Análisis documental y de información: dos componentes de un mismo proceso. ACIMED, 2.

Fernández, L. (2016). El uso didáctico y metodológico de las tabletas digitales en aulas de educación primaria y secundaria de Cataluña. Pixel-Bit. Revista de Medios y Educación, 48, 9-25. Recuperado de: http://dx.doi.org/10.12795/pixelbit.2016.i48.01

López García, N.J., De Moya, M.V., Cózar, R. Hernández, J.A. \& Hernández, J.R. (2016). Dispositivos android y educación musical: actividades de refuerzo y ampliación para $5^{\circ}$ y $6^{\circ}$ cursos de primaria. En L. Miranda, P. Alves \& C. Morais (Eds.), Estilos de aprendizaje: educación, tecnologías e innovación (pp. 1749-1761). Braganza: Instituto Politécnico de Braganza.

Massini, J. (2017). Mobile learning and cloud services beyond the textbook. Recommendations and guidelines for policy makers. Brussels: European Schoolnet-EUN Partnership AISBL. 
Matos, V., Cacheiro, M.L., Sánchez, C. \& González, J.M. (2012). Materiales audiovisuales. Una contribución a partir de los estilos de aprendizaje. En F. Guerra, R. García, N. González, P. Renés \& A. Castro (Coords.), Estilos de aprendizaje: investigaciones $y$ experiencias. Santander: Universidad de Cantabria. Recuperado de: https://bit.ly/2LF2Yaj

Montás, B. (2016). M-learning en los procesos de enseñanza-aprendizaje. Tecnología, Aprendizaje y Educación. Recuperado de: https://bit.ly/2IQj6YCl

Mosquera, I. (2018). M-learning: ventajas e inconvenientes del uso del móvil. UNIRrevista. Recuperado de: https://bit.ly/2OWXBED

Pajuelo, L. (2017). ¿Un móvil en clase? Educación 3.0. La Revista para el Aula del siglo XXI, 26, 22-31.

Peña, T. \& Pirela Morillo, J. (2007). La complejidad del análisis documental. Revista Información, Cultura y Sociedad, 16, 55-81. Recuperado de: https://bit.ly/2kziGYg

Robledo, J. (2012). Dispositivos móviles para el aprendizaje. Lo que usted necesita saber. Nueva York: Edutopia. George Lucas Educational Foundation.

Rubio, I. (12 de marzo de 2018). La probabilidad de fracaso escolar aumenta con el abuso de las nuevas tecnologías. El País. Recuperado de: https://bit.ly/2zDmTCl

Santiago, R., Díez, A. \& Navaridas, F. (2014). La taxonomía del aprendizaje a debate: del modelo de Bloom de los años 50 a la era del aprendizaje móvil. DIM. Revista Científica de Opinión y Divulgación, 29. Recuperado de: https://bit.ly/2IWSd5D

Shuler, C., Winters, N. \& West, M. (2013). El futuro del aprendizaje móvil. Implicaciones para la planificación y la formulación de políticas. París: UNESCO.

Recuperado

de: http://unesdoc.unesco.org/images/0021/002196/219637s.pdf

Stosic, L. \& Bogdanovic, M. (2013). M-learning. A new form of learning and education. IJCRSEE. International Journal of Cognitive Research in Science, Enginering and Education, 2 (1). Recuperado de: https://bit.ly/2ISN66q

UNESCO (2017). Supporting teachers with mobile technology. Lessons drawn from unesco projects in Mexico, Nigeria, Pakistan and Senegal. París: UNESCO. Recuperado de: http://unesdoc.unesco.org/images/0025/002515/251511e.pdf

Viñas, M. (2016). Tabletas en la educación: ventajas, retos, metodología y apps para facilitar el aprendizaje. Barcelona: Totemguard.

Vosloo, S. \& West M. (eds.) (2012a). Activando el aprendizaje móvil: temas globales. Políticas. París: UNESCO. Recuperado de: http://unesdoc.unesco.org/images/0021/002164/216451s.pdf 
Vosloo, S. \& West, M. (eds.) (2012b). Aprendizaje móvil para docentes. Temas globales. Docentes. París: UNESCO. Recuperado de: http://unesdoc.unesco.org/images/0021/002164/216452s.pdf

West, M. \& Vosloo, S. (coords.) (2013a), Directrices de la UNESCO para las políticas de aprendizaje móvil. París: UNESCO. Recuperado de: http://unesdoc.unesco.org/images/0021/002196/219662S.pdf

West, M. \& Vosloo, S. (coords.) (2013b). Aprendizaje móvil y políticas. Cuestiones clave. París: UNESCO. Recuperado de: http://unesdoc.unesco.org/images/0021/002176/217638s.pdf

Yot, C.R. \& Marcelo, C. (2015). ¿Despega el m-learning? Análisis de la disposición y hábitos de los usuarios. Pixel-Bit. Revista de Medios y Educación, 46, 205-218. Recuperado de: http://dx.doi.org/10.12795/pixelbit.2015.i46.13

\section{Para referenciar este artículo:}

López García, N. (2018). Políticas transnacionales sobre aprendizaje móvil y educación: una selección de textos relevantes. Edutec. Revista Electrónica de Tecnología Educativa, 65, 91-10. doi: https://doi.org/10.21556/edutec.2018.65.1133 